\title{
Impacto de las maniobras de reclutamiento alveolar en el diámetro de la vaina del nervio óptico en cirugía laparoscópica
}

\author{
Impact of alveolar recruitment maneuvers on optic nerve \\ sheath diameter in laparoscopic surgery
}

\author{
Alejandra Labra Nassar,* Darcy Danitza Marí Zapata, * \\ Gerardo Esteban Álvarez Reséndiz, ${ }^{\ddagger}$ Ronnie Aarón Ángeles de la Torre, * \\ Lizeth Hernández Escobar,* Christian García Ezquerro,* Ana de la Cajiga León* \\ Citar como: Labra NA, Marí ZDD, Álvarez RGE, Ángeles de la Torre RA, Hernández EL, García EC et al. \\ Impacto de las maniobras de reclutamiento alveolar en el diámetro de la vaina del nervio óptico en cirugía \\ laparoscópica. Acta Med Grupo Angeles. 2021; 19 (2): 172-179. https://dx.doi.org/10.35366/100438
}

\section{Resumen}

Las maniobras de reclutamiento alveolar pueden incrementar la presión intracraneal y disminuir la presión de perfusión cerebral; sin embargo, su uso se recomienda, ya que reduce las complicaciones pulmonares postoperatorias en cirugía laparoscópica en posición de Trendelenburg. Objetivo: Conocer el impacto de las maniobras de reclutamiento pulmonar en el diámetro de la vaina del nervio óptico, en cirugía laparoscópica en Trendelenburg. Material y métodos: Estudio experimental, prospectivo aleatorizado, comparativo. Se aleatorizaron 30 pacientes en dos grupos, grupo A: grupo control y grupo B: maniobra de reclutamiento alveolar. Pacientes en cirugía laparoscópica en posición de Trendelenburg; se midió el diámetro de la vaina del nervio óptico en diferentes tiempos. Resultado: El diámetro de la vaina del nervio óptico no presentó diferencia estadísticamente significativa entre el grupo control y el grupo maniobra durante las mediciones; sin embargo, mostró una diferencia significativa entre el valor basal y el valor previo a la emersión en ambos grupos $(p=0.001)$. Conclusión: El uso de una maniobra de reclutamiento alveolar que incrementa el PEEP de forma escalonada se puede aplicar de manera segura; sin embargo, se requieren más estudios con mayor número de muestra.

Palabras clave: Nervio óptico, presión intracraneal, reclutamiento alveolar, laparoscopia.

\section{Abstract}

Alveolar recruitment maneuvers can increase intracranial pressure and decrease cerebral perfusion pressure, however, their use is recommended as they reduce postoperative pulmonary complications in laparoscopic surgery in Trendelenburg position. Objective: To know the impact of pulmonary recruitment maneuvers on the diameter of the optic nerve sheath, in laparoscopic surgery in Trendelenburg position. Material and methods: Experimental, prospective randomized, comparative study. Thirty patients were randomized into two groups, group A: control group and group B: alveolar recruitment maneuver. Patients in laparoscopic surgery in Trendelenburg position, the diameter of the optic nerve was measured at different times. Result: The diameter of the optic nerve sheath did not show statistically significant difference between the control group and maneuver group during the measurements, however, we had a significant difference between the baseline value and the pre-emersion value in both groups $(p=0.001)$. Conclusion: The use of an alveolar recruitment maneuver by increasing PEEP in a scaled way can be applied safely, however, more studies with a larger sample number are required.

Keywords: Optic nerve, intracraneal pressure, alveolar recruitment, laparoscopy.

\footnotetext{
* Departamento de Anestesiología.

‡ Jefe del Departamento de Anestesiología.
}

Hospital Ángeles Lomas. Estado de México, México.
Correspondencia:

Alejandra Labra Nassar

Correo electrónico: alejandra_labra_9@hotmail.com

Aceptado: 17-04-2020.

www.medigraphic.com/actamedica 
Abreviaturas:

ASA $=$ American Society of Anesthesiologists

DVNO = Diámetro de la vaina del nervio óptico.

$\mathrm{ETCO}_{2}=$ Dióxido de carbono al final de la espiración.

$\mathrm{FiO}_{2} \%=$ Fracción inspirada de oxígeno.

LCR $=$ Líquido cefalorraquídeo.

MRA = Maniobras de reclutamiento alveolar.

PAM $=$ Presión arterial media.

PIC $=$ Presión intracraneal.

$\mathrm{PCO}_{2}=$ Presión parcial de dióxido de carbono.

PPC $=$ Presión perfusión cerebral.

PEEP $=$ Presión positiva al final de la espiración.

TOF $=$ Tren de cuatro.

$\mathrm{VC}=$ Volumen corriente.

\section{INTRODUCCIÓN}

La incidencia global de atelectasias durante la inducción de la anestesia general es de $85-90 \%{ }^{1}$ debido a una disminución de la capacidad pulmonar residual; ${ }^{2,3}$ sin embargo, las atelectasias especialmente en pulmones sanos se pueden prevenir con presión positiva, maniobras de reclutamiento alveolar (MRA), , 2,4,5 posicionamiento corporal, 6,7 fisioterapia $^{6}$ o entrenamiento muscular respiratorio..$^{8,9}$

Se ha comprobado que las MRA, cuyo objetivo es producir un aumento de la presión transpulmonar, ${ }^{10}$ mejoran la oxigenación intraoperatoria, disminuyen la necesidad de emplear $\mathrm{FiO}_{2}$ elevadas, reduciendo el colapso y complicaciones pulmonares postoperatorias, ${ }^{11}$ por lo que su uso debería implementarse de forma rutinaria después de la inducción anestésica seguida del empleo de presión positiva al final de la espiración (PEEP), salvo pacientes en los que estuviera contraindicado. ${ }^{1-5}$

El empleo de las MRA, niveles altos de PEEP, neumoperitoneo y la posición de Trendelenburg es controvertido, ya que puede aumentar la presión intracraneal (PIC) y disminuir la presión perfusión cerebral (PPC) $<65$ mmHg. ${ }^{12-15}$

La técnica estándar de oro para la medición de la PIC es la colocación de un catéter intraventricular; ${ }^{16}$ sin embargo, este método es invasivo y puede tener complicaciones como infección o hemorragia. ${ }^{17,18}$ Los métodos no invasivos de la presión intracraneal con el uso del ultrasonido como la medición del diámetro de la vaina del nervio óptico (DVNO) y el Doppler transcraneal son técnicas prometedoras en adultos y población pediátrica. ${ }^{19}$

La medición ecográfica del DVNO es una técnica confiable, rápida, segura y reproducible para la evaluación de la PIC. ${ }^{20-22}$ Se considera que el valor corte de 5.5-6.0 mm²3,24 o un aumento de $10 \%$ predice una presión intracraneal > $20 \mathrm{mmHg}$, con una sensibilidad y especificidad en el rango de $87-95 \%$ y de $79-100 \%$ respectivamente. ${ }^{21,25-27}$

El nervio óptico aparece como una estructura hipoecogénica detrás de la retina y del disco óptico, lo que proporciona un hito anatómico para el examen con ultrasonido.
La estructura hiperecogénica alrededor del nervio óptico representa el espacio subaracnoideo que está bordeado por la duramadre hipoecoica. ${ }^{28}$ Se deben realizar dos trazos: un primer vector vertical (A) desde la retina hasta la vaina que mida $3 \mathrm{~mm}$ y un segundo vector horizontal (B), perpendicular a éste pasando justo donde terminan los $3 \mathrm{~mm}$, midiendo la totalidad del diámetro de la vaina que se observa hipoecoico como se muestra en la Figura 1.

El objetivo de este estudio fue conocer el impacto de las de maniobras de reclutamiento pulmonar en el diámetro de la vaina del nervio óptico en pacientes para cirugía laparoscópica en Trendelenburg.

\section{MATERIAL Y MÉTODOS}

Diseño del estudio. Previa autorización del Comité Local de Investigación y Ética del Hospital Ángeles Lomas y una vez firmado el consentimiento informado, se realizó un estudio prospectivo, experimental, aleatorizado, en el cual se hicieron mediciones seriadas del DVNO. Se llevó a cabo dentro del servicio de las instalaciones de quirófanos correspondientes del Hospital Ángeles Lomas en el periodo marzo-junio de 2019.

Se incluyeron 34 pacientes adultos que fueran programados a cirugía laparoscópica en posición de Trendelenburg con los siguientes criterios de inclusión: estado físico del paciente I y II según la American Society of Anesthesiologists

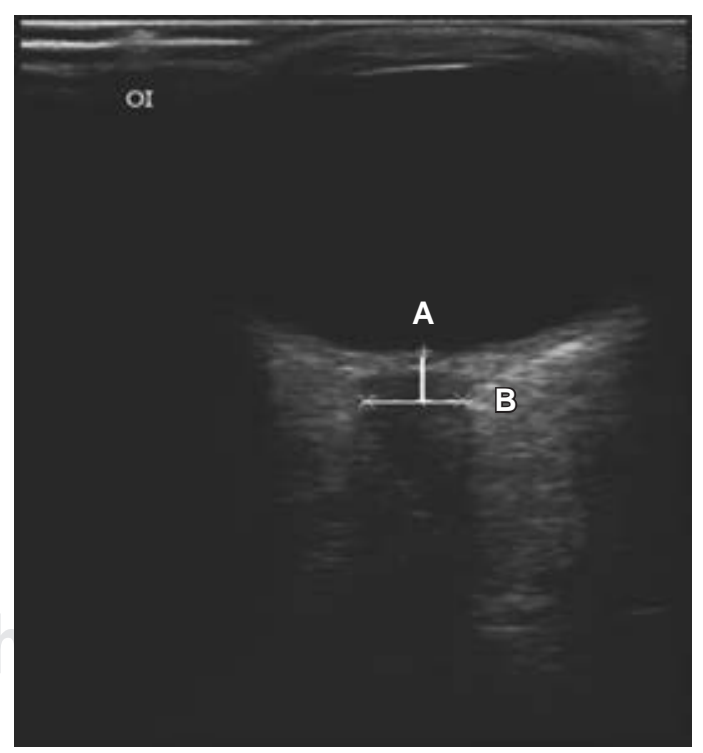

Figura 1: Vector A: se miden $3 \mathrm{~mm}$ iniciando en la retina en dirección vertical y vector $B$ : se traza una línea perpendicular al punto final de la línea A midiendo la longitud de toda la vaina del nervio óptico. Si es 5.5. $6.0 \mathrm{~mm}$ corresponde a una presión intracraneal $>20 \mathrm{mmHg}$. 
(ASA), con edad entre 20 y 60 años. Los criterios de exclusión fueron: pacientes con enfermedad pulmonar preoperatoria o preexistente significativa (asma activa, neumotórax, hemotórax, bullas pulmonares), pacientes con trauma craneoencefálico o hipertensión intracraneal, oftalmopatías (retinopatía diabética, glaucoma, desprendimiento de retina) cirugía oftalmológica dos meses previos, insuficiencia cardiaca descompensada, hipertensión pulmonar, estado de choque, índice de masa corporal $\geq 35$.

Los criterios de eliminación fueron: pacientes con presión arterial media (PAM) $<50$ refractaria durante el transanestésico, presencia de neumotórax, broncoespasmo durante el transanestésico, conversión a cirugía abierta o cancelación de maniobras de reclutamiento.

Tamaño de la muestra. El cálculo de muestra con el análisis de potencia realizado en el estudio piloto ${ }^{29}$ sugirió que se requiere un tamaño de muestra mínimo de 30 pacientes para detectar una diferencia de $0.5 \mathrm{~mm}$ (aproximadamente $10 \%$ de $4.9 \mathrm{~mm}$ ) en el diámetro de la vaina del nervio óptico media con una potencia de $80 \%$ y para detectar una diferencia relativa de $50 \%$ en el resultado primario a un nivel alfa bilateral de $p<0.05$ de importancia. Motivo por el que se realizará un tamaño de muestra de 33 pacientes esperando una tasa de abandono de alrededor de 10\%.

\section{Aleatorización}

La aleatorización para la asignación del grupo se efectuó con la técnica de sobre cerrado (asignación aleatoria simple manual), el cual se abrió al comienzo de la cirugía para recibir uno de los regímenes de ventilación. Grupo A (control): fracción inspirada de oxígeno $\left(\mathrm{FiO}_{2} \%\right)$ : $50 \%$, volumen corriente (VC) de $7 \mathrm{~mL} / \mathrm{kg}$ (peso corporal predicho), PEEP: $4 \mathrm{cmH}_{2} \mathrm{O}$, relación I:E 1:2, frecuencia respiratoria inicial de 10 respiraciones/min y luego se ajustó la ventilación (primero aumentando la frecuencia respiratoria y luego el VC en incrementos de $50 \mathrm{~mL}$ si fuera necesario) para mantener un dióxido de carbono al final de la espiración $\left(\mathrm{ETCO}_{2}\right)$ 30-35 $\mathrm{mmHg}$. Grupo B (maniobra de reclutamiento alveolar con PEEP escalonado): inicialmente con las mismas variables respiratorias de ventilación del grupo control, posterior a la colocación del neumoperitoneo (presión de inflado entre 13-15 $\mathrm{CmH}_{2} \mathrm{O}$ ) y posición Trendelenburg se aplicó la maniobra de reclutamiento pulmonar donde el VC y la relación I:E se mantendrá sin cambios mientras se reclutan, aumentando la PEEP gradualmente; con PEEP inicial de 4 $\mathrm{CmH}_{2} \mathrm{O}$ se incrementa a $10 \mathrm{cmH}_{2} \mathrm{O}$ (tres respiraciones) y por último $15 \mathrm{cmH}_{2} \mathrm{O}$ (10 respiraciones), tomando aproximadamente dos minutos en realizar una sola maniobra de reclutamiento alveolar. Se detuvo la MRA si la presión máxima de la vía aérea en cualquier punto excedía de 40 $\mathrm{cmH}_{2} \mathrm{O}$ y/o la PAM disminuía en más de $25 \%$ el valor de referencia, colocándose la PEEP inferior a la anterior. Después de las maniobras de reclutamiento, el VC se mantuvo sin cambios y la PEEP fija en $8 \mathrm{cmH}_{2} \mathrm{O}$.

Manejo anestésico

A la llegada al quirófano se abrió el sobre que programa el ventilador con los parámetros asignados en cada grupo. Se colocó monitoreo no invasivo (electrocardiograma de cinco derivaciones, toma de presión arterial no invasiva, pulsioxímetro, $\mathrm{ETCO}_{2}$ ), incluyendo BIS y tren de cuatro (TOF). Preoxigenación con flujo de oxígeno a $5 \mathrm{~L} / \mathrm{min}$, con un $\mathrm{FiO}_{2}: 100 \%$ durante tres a cinco minutos. La inducción se realizó con fentanilo: $3-5 \mu \mathrm{g} / \mathrm{kg}$, propofol: $2-2.5 \mathrm{mg} / \mathrm{kg}$, rocuronio: $0.6 \mathrm{mg} / \mathrm{kg}$, intubación con laringoscopia directa o videolaringoscopio según fuera el caso. Se cubrieron los ojos con Tegaderm. Mantenimiento anestésico con halogenado (desflurano, sevoflurano) para conservar un BIS en el rango de 40-60, bloqueo neuromuscular con TOF en cuenta de $0 \%$. Ventilación mecánica con máquina de anestesia DatexOhmeda Aestiva según los parámetros en cada grupo.

Para los episodios de desaturación arterial (pulsoximetría $\leq 92 \%$ ), un aumento transitorio en la fracción de oxígeno inhalado $\mathrm{FiO}_{2} \%$ se permitirá hasta $100 \%$.

Las decisiones sobre los cuidados del paciente durante los periodos intraoperatorio y postoperatorio, que incluyen la administración de líquidos, antibióticos profilácticos, elección de vasopresores en caso necesario y el manejo del dolor postoperatorio, se dejaron a discreción del anestesiólogo a cargo en cada procedimiento quirúrgico de acuerdo con la experiencia y la práctica clínica.

\section{Toma de mediciones}

Las mediciones fueron tomadas por un residente de tercer año de anestesiología con experiencia (más de 30 escaneos) con ultrasonido Terason uSmart 3300 utilizando un transductor lineal Array 7.5 MHz.

Se aplicó una capa de gel acústico, el transductor se colocó sobre el globo ocular con una presión ligera evitando que disminuya la velocidad del flujo sanguíneo en los vasos retroorbitales, y la duración fue menor de cinco minutos en cada ojo para evitar daños térmicos y de cavitación. ${ }^{30}$

Se realizaron tres mediciones transversales y tres mediciones verticales del diámetro de la vaina del nervio óptico en cada ojo en cada uno de los momentos de medición, registrando la media. Durante cada momento de medición se registraron las siguientes variables: frecuencia cardiaca, $\mathrm{PAM}$, saturación de oxígeno, $\mathrm{VC}$, volumen minuto, $\mathrm{ETCO}_{2}$, presión máxima de las vías aéreas, ventilación por minuto, compliance, distensibilidad estática, resistencia inspiratoria de la vía aérea, presión de distensión, distensibilidad 
Tabla 1: Características demográficas.

\begin{tabular}{|c|c|c|c|}
\hline \multirow[b]{2}{*}{$\begin{array}{l}\text { Características } \\
\text { demográficas }\end{array}$} & $\begin{array}{l}\text { Grupo control } \\
\qquad(N=15)\end{array}$ & $\begin{array}{c}\text { Grupo maniobra } \\
\qquad(\mathrm{N}=15)\end{array}$ & \multirow[b]{2}{*}{$p$} \\
\hline & n (\%) & $\mathrm{n}(\%)$ & \\
\hline $\begin{array}{l}\text { Edad (años), } \\
\text { media } \pm \text { DE }\end{array}$ & $54 \pm 11$ & $54 \pm 17$ & 0.9 \\
\hline Femenino & $7(46.7)$ & 5 (33.3) & 0.45 \\
\hline Masculino & $8(53.3)$ & $10(66.7)$ & 0.27 \\
\hline $\mathrm{IMC}$, media $\pm \mathrm{DE}$ & $28 \pm 6$ & $26 \pm 5$ & 0.54 \\
\hline ASA I & $2(13.3)$ & 5 (33.3) & 0.195 \\
\hline ASA II & $13(86.7)$ & $10(66.7)$ & 0.196 \\
\hline Gastroenterología & $11(73.3)$ & $8(53.3)$ & 0.159 \\
\hline Ginecología & 2 (13.3) & $1(6.7)$ & 0.24 \\
\hline Urología & $2(13.3)$ & $6(40)$ & 0.120 \\
\hline Cirugía robótica & $6(40)$ & 8 (53.3) & 0.46 \\
\hline HAS & $6(40)$ & $6(40)$ & 1.00 \\
\hline $\mathrm{DM} 2$ & $4(26.7)$ & $1(6.7)$ & 0.14 \\
\hline Hipotiroidismo & $3(20)$ & 0 & 0.06 \\
\hline Tabaquismo & $4(26.7)$ & $3(20)$ & 0.66 \\
\hline EPOC & 0 & 0 & 0.00 \\
\hline
\end{tabular}

Los datos se presentan en media \pm desviación estándar. No se observan cambios significativos entre los grupos.

IMC = índice de masa corporal; HAS = hipertensión arterial sistémica; DM2 = diabetes mellitus tipo II; EPOC = enfermedad pulmonar obstructiva crónica.

estática (VC / Pmeseta - PEEP), distensibilidad dinámica (VC / Pmáxima - PEEP), presión de distensión (Pmeseta PEEP), líquidos administrados, tiempo quirúrgico y tiempo anestésico.

Momentos de medición:

T0: cinco minutos después de la intubación orotraqueal.

T1: cinco minutos después del neumoperitoneo + posición de Trendelenburg.

T2: cinco minutos después de realizar la maniobra de reclutamiento en el grupo A o 10 minutos después del neumoperitoneo + posición de Trendelenburg en el grupo B.

T3: posterior al retiro del neumoperitoneo y en posición supina previa emersión.

Análisis estadístico. La estadística descriptiva incluye las medidas de tendencia central y dispersión, las variables categóricas expresadas como medidas de frecuencia absoluta y relativa y las variables numéricas como media y desviación estándar (DE). La sistematización de la información se realizó con el paquete STATA SE versión 11.1. y el nivel de significancia estadística aceptado se fijó como un valor de $p<0.05$.

\section{RESULTADOS}

De los 34 pacientes que cumplieron con los criterios de inclusión se descartaron tres, dos pacientes porque se tuvo que convertir el procedimiento en cirugía abierta, y uno porque se cancelaron las maniobras de reclutamiento. Se incluyeron respectivamente 15 pacientes en cada grupo con un total de 30 pacientes, $60 \%$ hombres, con una edad media de $54 \pm 11$ años e índice de masa corporal media de $26.97 \pm 5.2 \mathrm{~kg} / \mathrm{m}^{2}$. El riesgo quirúrgico fue ASA I y II en $23 \%$ y $76.7 \%$, respectivamente. No se encontraron diferencias estadísticamente significativas en las variables demográficas. Los datos se detallan en la Tabla 1.

\section{Medición basal y mediciones seriadas del diámetro de la vaina del nervio óptico}

No se observó diferencia estadísticamente significativa en la medición del DVNO al inicio (T0, p = 0.19), al cambio de posición $(\mathrm{T} 1, \mathrm{p}=0.507)$, durante la maniobra de reclutamiento alveolar $(\mathrm{T} 2, \mathrm{p}=0.507)$ y al retiro de neumoperitoneo ( $\mathrm{T} 3, \mathrm{p}=0.336)$ entre el grupo control con el grupo maniobra (Tabla 2).

Sin embargo, pudimos observar diferencia significativa entre T0 y T3 en el grupo control $(p=0.001)$ y en el grupo maniobra $(p=0.001)$, aunque sin impacto clínico (Figuras 2 y 3 ).

\section{Riesgo de hipertensión intracraneal}

El riesgo de hipertensión intracraneal se define como DVNO > $0.55 \mathrm{~cm}$ : se observó una proporción similar de

\begin{tabular}{|c|c|c|c|}
\hline \multicolumn{4}{|c|}{$\begin{array}{l}\text { Tabla 2: Mediciones del diámetro } \\
\text { de la vaina del nervio óptico. }\end{array}$} \\
\hline \multirow{2}{*}{$\begin{array}{l}\text { DVNO } \\
(\mathrm{cm})\end{array}$} & $\begin{array}{l}\text { Grupo control } \\
\qquad(N=15)\end{array}$ & $\begin{array}{l}\text { Grupo maniobra } \\
\qquad(\mathrm{N}=15)\end{array}$ & \multirow[b]{2}{*}{$p$} \\
\hline & \multicolumn{2}{|c|}{ Media $\pm D E$} & \\
\hline TO & $0.47 \pm 0.06$ & $0.44 \pm 0.06$ & 0.19 \\
\hline $\mathrm{T} 1$ & $0.50 \pm 0.06$ & $0.49 \pm 0.06$ & 0.507 \\
\hline $\mathrm{T} 2$ & $0.50 \pm 0.06$ & $0.49 \pm 0.06$ & 0.507 \\
\hline T3 & $0.50 \pm 0.06$ & $0.48 \pm 0.07$ & 0.336 \\
\hline
\end{tabular}


pacientes con datos indirectos de hipertensión intracraneal en el grupo de maniobra con $6.7 \%$ vs $20 \%(p=0.28)$ en el grupo control, una menor proporción de sujetos con hipertensión intracraneal en el grupo en el que se realizó la maniobra de reclutamiento alveolar con un $\mathrm{RR}=0.33$ (IC 95\%: 0.039-2.85, [p = 0.28]).

\section{Comparación de las variables de la ventilación mecánica entre los grupos}

Durante la maniobra de reclutamiento alveolar (T2) se encontraron diferencias estadísticamente significativas con aumento de la presión media, presión meseta, compliancia pulmonar, distensibilidad dinámica y distensibilidad estática, y disminución de la resistencia de la vía aérea y presión de distensión. Los resultados se detallan en la Tabla 3.

\section{DISCUSIÓN}

La medición del diámetro de la vaina del nervio óptico (DVNO) con ultrasonido es una medición no invasiva de la presión intracraneal con una sensibilidad y especificidad de $95.8 \%$ y $80.4 \%$ respectivamente, ${ }^{31}$ puede incluirse como monitoreo de rutina en pacientes en quienes la medición de la presión intracraneal invasiva tenga mayor riesgo que beneficio. La posición de Trendelenburg más la colocación de neumoperitoneo con $\mathrm{CO}_{2}$ y la ejecución de una maniobra de reclutamiento alveolar son factores de riesgo de incrementar la presión intracraneal. Se ha comprobado que la posición de Trendelenburg por sí sola

Figura 2: Gráfica de cajas que demuestra el DVNO en el grupo control, comparando T0 con T3 con una diferencia estadísticamente significativa $(p=0.001)$.

DVNO = diámetro de la vaina del nervio óptico; To = cinco minutos después de la intubación orotraqueal; T3 = posterior al retiro del neumoperitoneo y en posición supina previa emersión.

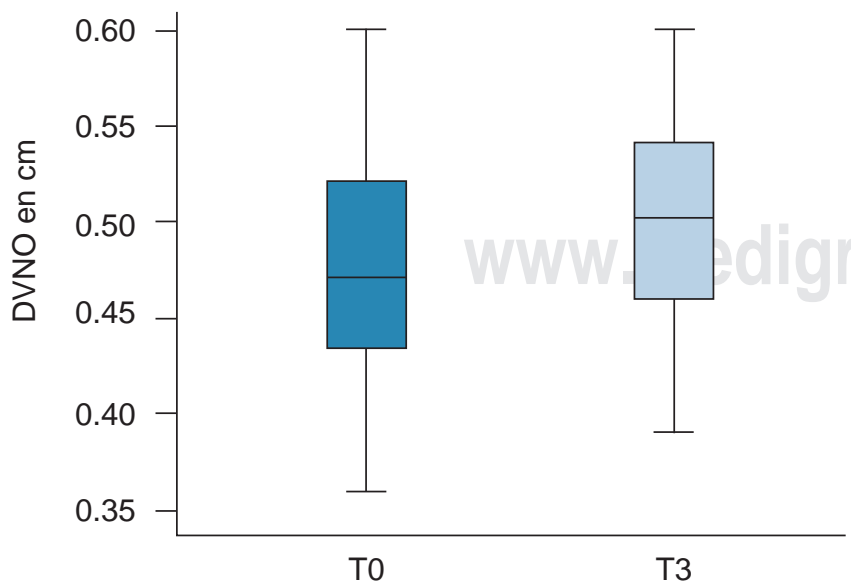

Figura 3: Gráfica de cajas que demuestra el DVNO en el grupo maniobra, comparando T0 con T3 con una diferencia estadísticamente significativa $(p=0.001)$.

DVNO = diámetro de la vaina del nervio óptico; T0 = cinco minutos después de la intubación orotraqueal; T3 = posterior al retiro del neumoperitoneo y en posición supina previa emersión.

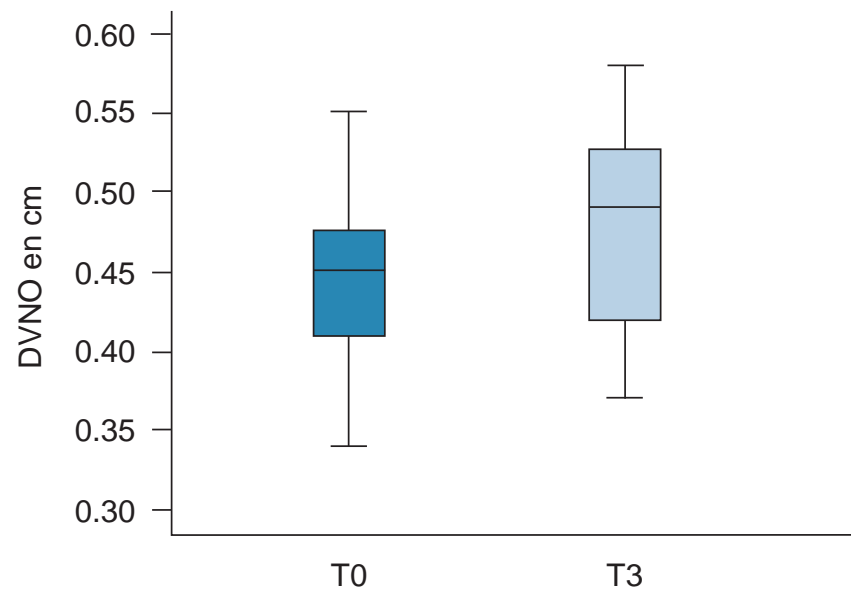

puede incrementar la presión intracraneal de 9 a $13 \mathrm{mmHg}$ en pacientes neurológicos con monitoreo directo de la presión intracraneal. ${ }^{32}$ Este incremento se debe a un aumento súbito de la presión intraabdominal, elevación de la presión intratorácica, hipercapnia y aumento de la presión venosa central disminuyendo así el drenaje venoso yugular. En este caso controlamos el $\mathrm{ETCO}_{2}$ modificando el volumen minuto (frecuencia respiratoria y volumen corriente), si no se hubiera modificado este parámetro ventilatorio durante la insuflación, la vasodilatación cerebral inducida por $\mathrm{CO}_{2}$ probablemente hubiera contribuido a un aumento del DVNO. En nuestro estudio realizamos una maniobra de reclutamiento con un incremento de la PEEP de $5 \mathrm{cmH}_{2} \mathrm{O}$ en $5 \mathrm{cmH}_{2} \mathrm{O}$ hasta $15 \mathrm{cmH}_{2} \mathrm{O}$ durante 10 inspiraciones después de 10 minutos de colocar al paciente en posición de Trendelenburg con neumoperitoneo, observando que no se obtuvo diferencia en el DVNO entre el grupo control $0.49 \pm 0.06$ vs grupo maniobra $0.50 \pm 0.05 \mathrm{~cm}(p=0.5)$, al igual que en la comparación del grupo maniobra antes de realizar la manobra de reclutamiento $0.49 \pm 0.06$ y posterior a la misma $0.49 \pm 0.06$, siendo nuestros resultados similares a los de Chin JH y colaboradores y Ann Hee You y su equipo; sin embargo, ellos no realizaron una maniobra de reclutamiento, aleatorizaron un grupo control y un grupo con PEEP $8 \mathrm{cmH}_{2} \mathrm{O}$ y $5 \mathrm{cmH}_{2} \mathrm{O}$ respectivamente en cada estudio. ${ }^{29,33}$

En estudios previos en prostatectomías por robot donde midieron el DVNO, asociaron que un aumento de la PAM de $10 \mathrm{mmHg}$ el DVNO incrementaba $0.023 \mathrm{~mm},{ }^{22}$ otro estudio demostró un incremento de 12.5\% del DVNO 10 
y 30 minutos después de la posición de Trendelenburg y neumoperitoneo. ${ }^{34}$ Un estudio de 21 pacientes reveló que tres minutos después de la posición de Trendelenburg y neumoperitoneo el DVNO incrementó $0.4 \mathrm{~mm} .{ }^{35}$ Se ha asociado que el desflurano en comparación con el sevoflurano tiene un incremento mayor del flujo sanguíneo cerebral elevando así en mayor grado la PIC. ${ }^{36}$ Fernando Dip y colaboradores detectaron un incremento de $1 \mathrm{~mm}$ del DVNO $(p \leq 0.0001)^{37}$ en nuestro estudio pudimos observar diferencia significativa entre T0 y T3 en el grupo control ( $p=0.001)$ y maniobra $(p=0.001)$, aunque clínicamente sin impacto a pesar de que en otros estudios no se observaron diferencias significativas en el DVNO. ${ }^{29,33,38}$

Encontramos un paciente en el grupo de maniobra que mostró hipertensión intracraneal con un DVNO $>0.55 \mathrm{~cm}$, con un RR = 0.33 (IC 95\%: 0.039-2.85 [p = 0.28]). En el grupo control se presentaron tres casos, por lo que podemos deducir que la maniobra de reclutamiento alveolar no tuvo ninguna repercusión a nivel cerebral. Este incremento del DVNO en cuatro pacientes se presentó en cirugía de robot con una duración quirúrgica de $>180$ minutos.

Al realizar la comparación de las variables de la ventilación mecánica entre los grupos, se detectaron diferencias estadísticamente significativas; aunque la presión máxima y la presión meseta fueron mayores en el momento que se realizó la MRA, podemos observar que la presión de distensión es menor durante la MRA $13 \pm 4$ vs $20 \pm 3$ ( $p$ $\leq 0.001$ ). Pacientes sometidos a cirugía con presión de distensión elevada $\left(>15 \mathrm{cmH}_{2} \mathrm{O}\right.$ ) se asocian con más complicaciones pulmonares postoperatorias. ${ }^{39,40}$ Nuestra maniobra de reclutamiento alveolar que consistió en incrementar de manera gradual el PEEP hasta llegar a 15 $\mathrm{CmH}_{2} \mathrm{O}$ de PEEP, disminuyó de forma significativa la presión de distensión e incrementó la compliance pulmonar con una diferencia significativa en el grupo A: $27 \pm 9$ vs $39 \pm$ 16 en el grupo $B(p=024)$.
El reclutamiento alveolar con aumentos escalonados de la PEEP que incrementa la presión inspiratoria durante 5-10 respiraciones es una estrategia efectiva para mejorar la oxigenación arterial y la presión de distensión; ${ }^{5}$ esta maniobra fue descrita por Whalen y colaboradores y Sprung y su equipo. ${ }^{41,42}$ Empleamos esta MRA en nuestro estudio, ya que mantiene el alveolo abierto generando menor riesgo de barotrauma; sin embargo, sólo se realizó 10 minutos posterior a la insuflación del neumoperitoneo y la posición de Trendelenburg que es el momento de mayor riesgo de generación de atelectasias, las cuales provocan complicaciones pulmonares postoperatorias.

\section{CONCLUSIÓN}

En pacientes con ASA I y II no se encontró diferencia significativa en el DVNO en cirugía laparoscópica en posición de Trendelenburg al realizar una maniobra de reclutamiento alveolar. Aunque se observó una diferencia estadísticamente significativa entre el valor basal y el valor previo a la emersión, ésta no posee relevancia clínica.

Estos resultados sugieren que las maniobras de reclutamiento alveolar que incrementan el PEEP de forma escalonada se pueden aplicar de manera segura en cirugía laparoscópica en posición de Trendelenburg disminuyendo así el riesgo de complicaciones pulmonares; sin embargo, no se recomiendan en pacientes con enfermedad ocular y patología cerebral preexistentes, ya que pueden tener mayor riesgo de hipertensión intracraneal debido a que la autorregulación se encuentra alterada y la capacidad para mantener estable el flujo sanguíneo está disminuida, lo que incrementa el riesgo de isquemia.

Aunque se observó una diferencia estadísticamente significativa entre el valor basal (T0) y el valor previo a la emersión (T3) $(p=0.01)$, ésta no posee relevancia clínica.

Tabla 3: Variables de la ventilación mecánica.

\begin{tabular}{|c|c|c|c|}
\hline \multirow[b]{2}{*}{ T2 } & Grupo control (N = 15) & \multicolumn{2}{|c|}{ Grupo maniobra $(\mathrm{N}=15)$} \\
\hline & \multicolumn{2}{|c|}{ Media $\pm \mathrm{DE}$} & $p$ \\
\hline Presión máxima $\left(\mathrm{cmH}_{2} \mathrm{O}\right)$ & $28 \pm 4$ & $31 \pm 3$ & 0.05 \\
\hline Presión media $\left(\mathrm{cmH}_{2} \mathrm{O}\right)$ & $13 \pm 2$ & $18 \pm 5$ & 0.003 \\
\hline Presión meseta $\left(\mathrm{cmH}_{2} \mathrm{O}\right)$ & $24 \pm 3$ & $28 \pm 4$ & 0.009 \\
\hline Compliancia $\left(\mathrm{mL} / \mathrm{cmH}_{2} \mathrm{O}\right)$ & $27 \pm 9$ & $39 \pm 16$ & 0.024 \\
\hline Resistencia de vía aérea $\left(\mathrm{cmH}_{2} \mathrm{O} / \mathrm{L} / \mathrm{s}\right)$ & $17 \pm 6$ & $10 \pm 3$ & 0.001 \\
\hline Presión de distensión $\left(\mathrm{cmH}_{2} \mathrm{O}\right)^{2}$ & $20 \pm 3$ & $13 \pm 4$ & $<0.001$ \\
\hline Distensibilidad dinámica $\left(\mathrm{mL} / \mathrm{cmH}_{2} \mathrm{O}\right)$ & $20 \pm 5$ & $29 \pm 7$ & $<0.001$ \\
\hline Distensibilidad estática $\left(\mathrm{mL} / \mathrm{cmH}_{2} \mathrm{O}\right)$ & $24 \pm 7$ & $38 \pm 19$ & 0.012 \\
\hline
\end{tabular}


Se necesitan más estudios clínicos, con mayor número de muestra que tengan como objetivo dilucidar el nivel crítico de las MRA, PEEP y los efectos de otras estrategias en pacientes con colapso pulmonar en cirugía laparoscópica.

\section{REFERENCIAS}

1. Hartland BL, Newell TJ, Damico N. Alveolar recruitment maneuvers under general anesthesia: a systematic review of the literature. Respir Care. 2015; 60 (4): 609-620.

2. Rusca M, Proietti S, Schnyder P, Frascarolo P, Hedenstierna G, Spahn DR et al. Prevention of atelectasis formation during induction of general anesthesia. Anesth Analg. 2003; 97 (6): 1835-1839.

3. García-Fernández J, Canfrán S, de Segura IA, Suarez-Sipmann F, Aguado D, Hedenstierna G. Pressure safety range of barotrauma with lung recruitment manoeuvres: a randomised experimental study in a healthy animal model. Eur J Anaesthesiol. 2013; 30 (9): 567-574.

4. Chiumello D, Chevallard G, Gregoretti C. Non-invasive ventilation in postoperative patients: a systematic review. Intensive Care Med. 2011; 37 (6): 918-929.

5. Rothen HU, Sporre B, Engberg G, Wegenius G, Hedenstierna G. Re-expansion of atelectasis during general anaesthesia: a computed tomography study. Br J Anaesth. 1993; 71 (6): 788-795.

6. Raoof S, Chowdhrey N, Raoof S, Feuerman M, King A, Sriraman R et al. Effect of combined kinetic therapy and percussion therapy on the resolution of atelectasis in critically ill patients. Chest. 1999; 115 (6): 1658-1666.

7. Staudinger T, Bojic A, Holzinger U, Meyer B, Rohwer M, Mallner F et al. Continuous lateral rotation therapy to prevent ventilator-associated pneumonia. Crit Care Med. 2010; 38 (2): 486-490.

8. Stock MC, Downs JB, Gauer PK, Alster JM, Imrey PB. Prevention of postoperative pulmonary complications with CPAP, incentive spirometry, and conservative therapy. Chest. 1985; 87 (2): 151-157.

9. Hulzebos EH, Helders PJ, Favié NJ, De Bie RA, Brutel de la Riviere A, Van Meeteren NL. Preoperative intensive inspiratory muscle training to prevent postoperative pulmonary complications in highrisk patients undergoing $C A B G$ surgery: a randomized clinical trial. JAMA. 2006; 296 (15): 1851-1857.

10. Tusman G, Bohm SH, Vazquez de Anda GF, do Campo JL, Lachmann B. 'Alveolar recruitment strategy' improves arterial oxygenation during general anaesthesia. Br J Anaesth. 1999; 82 (1): 8-13.

11. Young CC, Harris EM, Vacchiano C, Bodnar S, Bukowy B, Elliott RRD et al. Lung-protective ventilation for the surgical patient: international expert panel-based consensus recommendations. Br J Anaesth. 2019; 123 (6): 898-913.

12. Chesnut RM. Care of central nervous system injuries. Surg Clin North Am. 2007; 87 (1): 119-156, vii.

13. Bein T, Kuhr LP, Bele S, Ploner F, Keyl C, Taeger K. Lung recruitment maneuver in patients with cerebral injury: effects on intracranial pressure and cerebral metabolism. Intensive Care Med. 2002; 28 (5): 554-558.

14. Bloomfield GL, Ridings PC, Blocher CR, Marmarou A, Sugerman HJ. A proposed relationship between increased intra-abdominal, intrathoracic, and intracranial pressure. Crit Care Med. 1997; 25 (3): 496-503.

15. Citerio G, Vascotto E, Villa F, Celotti S, Pesenti A. Induced abdominal compartment syndrome increases intracranial pressure in neurotrauma patients: a prospective study. Crit Care Med. 2001; 29 (7): 1466-1471.

16. Bratton SL, Chestnut RM, Ghajar J, McConnell Hammond FF, Harris $\mathrm{OA}$, Hartl R et al. Guidelines for the management of severe traumatic brain injury. VI. Indications for intracranial pressure monitoring. J Neurotrauma. 2007; 24 Suppl 1: S37-S44.
17. Hoefnagel D, Dammers R, Ter Laak-Poort MP, Avezaat CJ. Risk factors for infections related to external ventricular drainage. Acta Neurochir (Wien). 2008; 150 (3): 209-214; discussion 214.

18. Binz DD, Toussaint LG 3rd, Friedman JA. Hemorrhagic complications of ventriculostomy placement: a meta-analysis. Neurocrit Care. 2009; 10 (2): 253-256.

19. Albin MS. Measuring the ICP in neonates and infants noninvasively is also important. J Neurosurg Anesthesiol. 2014; 26 (4): 407.

20. Robba C, Cardim D, Tajsic T, Pietersen J, Bulman M, Donnelly J et al. Ultrasound non-invasive measurement of intracranial pressure in neurointensive care: A prospective observational study. PLoS Med. 2017; 14 (7): e1002356.

21. Geeraerts T, Merceron S, Benhamou D, Vigué B, Duranteau J. Non-invasive assessment of intracranial pressure using ocular sonography in neurocritical care patients. Intensive Care Med. 2008; 34 (11): 2062-2067.

22. Whiteley JR, Taylor J, Henry M, Epperson TI, Hand WR. Detection of elevated intracranial pressure in robot-assisted laparoscopic radical prostatectomy using ultrasonography of optic nerve sheath diameter. J Neurosurg Anesthesiol. 2015; 27 (2): 155-159.

23. Goel RS, Goyal NK, Dharap SB, Kumar M, Gore MA. Utility of optic nerve ultrasonography in head injury. Injury. 2008; 39 (5): 519-524.

24. Lambert DM, Marceau S, Forse RA. Intra-abdominal pressure in the morbidly obese. Obes Surg. 2005; 15 (9): 1225-1232.

25. Soldatos T, Chatzimichail K, Papathanasiou M, Gouliamos A. Optic nerve sonography: a new window for the non-invasive evaluation of intracranial pressure in brain injury. Emerg Med J. 2009; 26 (9): 630-634.

26. Watanabe A, Horikoshi T, Uchida M, Ishigame K, Kinouchi H. Decreased diameter of the optic nerve sheath associated with CSF hypovolemia. AJNR Am J Neuroradiol. 2008; 29 (5): 863-864.

27. Kimberly HH, Shah S, Marill K, Noble V. Correlation of optic nerve sheath diameter with direct measurement of intracranial pressure. Acad Emerg Med. 2008; 15 (2): 201-204.

28. Killer HE, Laeng HR, Flammer J, Groscurth P. Architecture of arachnoid trabeculae, pillars, and septa in the subarachnoid space of the human optic nerve: anatomy and clinical considerations. $\mathrm{Br} J$ Ophthalmol. 2003; 87 (6): 777-781.

29. Chin JH, Kim WJ, Lee J, Han YA, Lim J, Hwang JH et al. Effect of positive end-expiratory pressure on the sonographic optic nerve sheath diameter as a surrogate for intracranial pressure during robotassisted laparoscopic prostatectomy: a randomized controlled trial. PLoS One. 2017; 12 (1): e0170369.

30. Tranquart F, Berges O, Koskas P, Arsene S, Rossazza C, Pisella PJ et al. Color Doppler imaging of orbital vessels: personal experience and literature review. J Clin Ultrasound. 2003; 31 (5): 258-273.

31. Raffiz M, Abdullah JM. Optic nerve sheath diameter measurement: a means of detecting raised ICP in adult traumatic and non-traumatic neurosurgical patients. Am J Emerg Med. 2017; 35 (1): 150-153.

32. Mavrocordatos P, Bissonnette B, Ravussin P. Effects of neck position and head elevation on intracranial pressure in anaesthetized neurosurgical patients: preliminary results. J Neurosurg Anesthesiol. 2000; 12 (1): 10-14.

33. You AH, Song Y, Kim DH, Suh J, Baek JW, Han DW. Effects of positive end-expiratory pressure on intraocular pressure and optic nerve sheath diameter in robot-assisted laparoscopic radical prostatectomy: A randomized, clinical trial. Medicine (Baltimore). 2019; 98 (14): e15051.

Kim MS, Bai SJ, Lee JR, Choi YD, Kim YJ, Choi SH. Increase in intracranial pressure during carbon dioxide pneumoperitoneum with steep Trendelenburg positioning proven by ultrasonographic measurement of optic nerve sheath diameter. J Endourol. 2014; 28 (7): 801-806.

35. Chin JH, Seo H, Lee EH, Lee J, Hong JH, Hwang JH et al. Sonographic optic nerve sheath diameter as a surrogate measure for intracranial pressure in anesthetized patients in the Trendelenburg position. BMC Anesthesiol. 2015; 15: 43.

36. Holmstrom A, Akeson J. Desflurane increases intracranial pressure more and sevoflurane less than isoflurane in pigs subjected to intracranial hypertension. J Neurosurg Anesthesiol. 2004; 16 (2): 136-143. 
37. Dip F, Nguyen D, Rosales A, Sasson M, Lo Menzo E, Szomstein S et al. Impact of controlled intraabdominal pressure on the optic nerve sheath diameter during laparoscopic procedures. Surg Endosc. 2016; 30 (1): 44-49.

38. Verdonck P, Kalmar AF, Suy K, Geeraerts T, Vercauteren M, Mottrie A et al. Optic nerve sheath diameter remains constant during robot assisted laparoscopic radical prostatectomy. PLoS One. 2014; 9 (11): e111916.

39. Kacmarek RM, Villar J. Lung-protective ventilation in the operating room: individualized positive end-expiratory pressure is needed! Anesthesiology. 2018; 129 (6): 1057-1059.

40. Neto AS, Hemmes SN, Barbas CS, Beiderlinden M, FernandezBustamante A, Futier E et al. Association between driving pressure and development of postoperative pulmonary complications in patients undergoing mechanical ventilation for general anaesthesia: a meta-analysis of individual patient data. Lancet Respir Med. 2016; 4 (4): 272-280.

41. Whalen FX, Gajic O, Thompson GB, Kendrick ML, Que FL, Williams $\mathrm{BA}$ et al. The effects of the alveolar recruitment maneuver and positive end-expiratory pressure on arterial oxygenation during laparoscopic bariatric surgery. Anesth Analg. 2006; 102 (1): 298305.

42. Sprung J, Whalen FX, Comfere T, Bosnjak ZJ, Bajzer Z, Gajic O et al. Alveolar recruitment and arterial desflurane concentration during bariatric surgery. Anesth Analg. 2009; 108 (1): 120-127. 